\title{
N-(Phenylthio)-e-caprolactam: A New Promoter for the Activation of Thioglycosides
}

\author{
Sergio G. Durón, Tülay Polat, and Chi-Huey Wong* \\ Department of Chemistry and the Skaggs Institute for Chemical Biology, The Scripps \\ Research Institute, 10550 North Torey Pines Road, La Jolla, CA 92037
}

\section{Supporting Material}

General Procedures. All reactions were performed in flame-dried modified Schlenk (Kjeldahl shape) flasks fitted with a glass stopper or rubber septa under a positive pressure of argon, unless otherwise noted. Air- and moisture-sensitive liquids and solutions were transferred via syringe or stainless steel cannula. All saccharides and promoters were pre-dried by azeotropic removal of water using toluene. Organic solutions were concentrated by rotary evaporation below $30{ }^{\circ} \mathrm{C}$ at ca. 25 Torr. Flash column chromatography was performed employing Silica Gel 60 Sorbent $(40-63 \mu \mathrm{m}$, 230-400 mesh). Thin-layer chromatography (analytical and preparative) was performed using glass plates pre-coated to a depth of $0.25 \mathrm{~mm}$ with $230-400$ mesh silica gel impregnated with a fluorescent indicator $(254 \mathrm{~nm})$ and visualized under UV $(254 \mathrm{~nm})$ and/or by staining with acidic ceric ammonium molybdate.

Materials. Dichloromethane and Toluene were freshly distilled from calcium hydride at 760 Torr. Trifluroromethanesulfonic anhydride was purchased from Aldrich and distilled from phosphorous pentoxide. Triethylamine was purchased from Aldrich and used without further purification. Tri-t-butylpyrimidine was purchased from Lakeview Synthesis and used without further purification. Molecular sieves (MS) (type AW-300) were crushed and freshly flame activated before every use. $N$-(thiophenyl)- $\varepsilon-$ caprolactam (1) was either synthesized or purchased from Aldrich (rare chemical inventory).

Instrumentation. Proton and carbon-13 nuclear magnetic resonance $\left({ }^{1} \mathrm{H}\right.$ NMR or ${ }^{13} \mathrm{C}$ NMR) spectra were recorded on a Varian 400, a Bruker DRX-500 (500 MHz) 500, or a Bruker DRX-600 (600 MHz) spectrometer at $20{ }^{\circ} \mathrm{C}$. Chemical shifts are expressed in parts per million ( $\delta$ scale) downfield from tetramethylsilane and are referenced to either residual protium in the NMR solvent $(\mathrm{CHCl} 3: \delta=7.26 \mathrm{ppm})$ or to tetramethylsilane $(\delta=$ $0 \mathrm{ppm}$ ) if the residual protium of the solvent is indistinguishable. Data are presented as follows: chemical shift, multiplicity $(\mathrm{s}=$ singlet, $\mathrm{d}=$ doublet, $\mathrm{t}=$ triplet, $\mathrm{m}=$ multiplet and/or multiple resonances), integration, coupling constant in Hertz (Hz). Carbon-13 NMR were obtained using the same instruments and were calibrated with $\mathrm{CDCl} 3(\delta=$ $77.00 \mathrm{ppm}$ ). MALDI high-resolution mass spectrometry was performed on a IonSpec Ultima FTMS. 


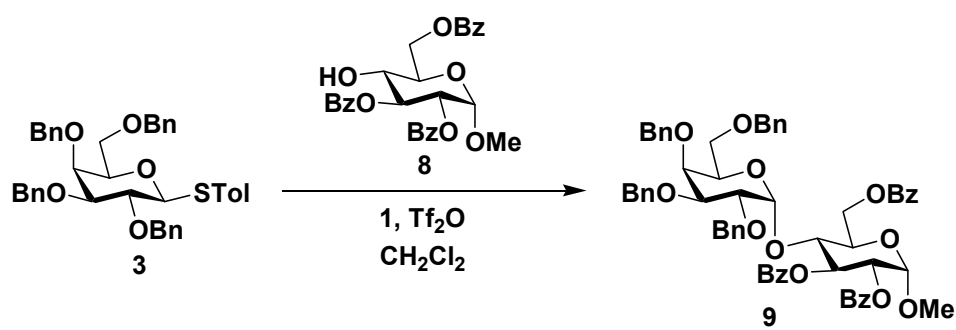

\section{Methyl-2,3,6-tri- $O$-benzoyl-4- $O$-[2,3,4,6-tetra- $O$-benzyl- $\alpha$-D-galactopyranosyl]- $\alpha$-D-}

glucopyranoside (9): A solution of $\mathbf{3}(50.5 \mathrm{mg}, 0.0781 \mathrm{mmol}), \mathbf{8}(37 \mathrm{mg}, 0.0731 \mathrm{mmol})$, $N$-(thiophenyl)- $\varepsilon$-caprolactam (1) (18 $\mathrm{mg}, 0.0813 \mathrm{mmol})$, and flame activated AW-300 MS were stirred in dry dichloromethane $(2 \mathrm{ml})$ for $0.5 \mathrm{~h}$ at ambient temperature. The reaction mixture was then cooled to $-45^{\circ} \mathrm{C}\left(\mathrm{CH}_{3} \mathrm{CN} /\right.$ Dry Ice), stirred for $5 \mathrm{~min}$ and then followed by injection of trifluoromethanesulfonic anhydride (16 $\mu \mathrm{L}, 0.0945 \mathrm{mmol})$. The reaction mixture was stirred for $0.5 \mathrm{~h}$ at $-45^{\circ} \mathrm{C}$, then at ambient temperature for $0.5 \mathrm{~h}$. The solution was quenched with triethylamine $(150 \mu \mathrm{L})$, filtered and concentrated. The crude mixture was directly purified by silica gel flash column chromatography (hexane/EtOAc, 5:1) to afford $9(67 \mathrm{mg}, 89 \%)$ as a white foam.

9: ${ }^{1} \mathrm{H}$ NMR $\left(500 \mathrm{MHz}, \mathrm{CDCl}_{3}\right) \delta$ 8.10-8.05 (m, 2H), 8.00-7.95 (m, 4H), 7.62-7.08 (m, $29 \mathrm{H}), 6.19(\mathrm{t}, 1 \mathrm{H}, J=9.1 \mathrm{~Hz}), 5.22(\mathrm{dd}, 1 \mathrm{H}, J=10.3,3.8 \mathrm{~Hz}), 5.16(\mathrm{~d}, 1 \mathrm{H}, J=3.6 \mathrm{~Hz}$, $\left.\mathrm{H}^{\prime}-1\right), 5.13$ (d, 1H, J=3.5 Hz, H-1), 4.82-4.78 (m, 2H), 4.65-4.59 (m, 3H), 4.42 (d, 1H, $J$ $=11.4 \mathrm{~Hz}), 4.33-4.21(\mathrm{~m}, 4 \mathrm{H}), 4.15(\mathrm{~d}, 1 \mathrm{H}, J=11.7 \mathrm{~Hz}), 4.05(\mathrm{~d}, 1 \mathrm{H}, J=12.1 \mathrm{~Hz}), 4.00$ $(\mathrm{t}, 1 \mathrm{H}, J=6.6 \mathrm{~Hz}), 3.91-3.88(\mathrm{~m}, 2 \mathrm{H}), 3.82(\mathrm{dd}, 1 \mathrm{H}, J=9.9,3.6 \mathrm{~Hz}), 3.47-3.41(\mathrm{~m}, 1 \mathrm{H})$, $3.42(\mathrm{~s}, 3 \mathrm{H}), 3.36(\mathrm{dd}, 1 \mathrm{H}, J=8.9,5.8) ;{ }^{13} \mathrm{C} \mathrm{NMR}\left(125 \mathrm{MHz}, \mathrm{CDCl}_{3}\right)$ 166.1, 166.0, $165.5,138.4,138.2,137.9,133.3,133.1,132.9,129.9,129.8,129.7,129.6,128.5,128.5$, $128.4,128.3,128.2,128.1,128.0,127.7,127.6,127.5,127.4,98.9$ (C'-1), 96.7 (C-1), 
78.7, 75.3, 74.9, 74.7, 74.5, 73.2, 73.1, 72.9, 72.2, 72.1, 70.6, 68.6, 68.5, 63.4, 55.3;

HRMS (MALDI-FTMS) $m / z:(\mathrm{M}+\mathrm{Na})^{+}$calc for $\mathrm{C}_{62} \mathrm{H}_{60} \mathrm{O}_{14} 1051.3875$, found 1051.3881 .
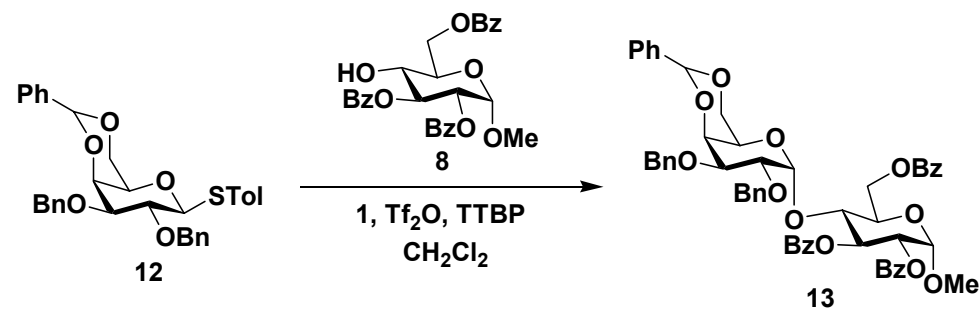

\section{Methyl-2,3,6-tri- $O$-benzoyl-4- $O$-[2,3-di- $O$-benzyl-4,6- $O$-benzylidene- $\alpha$-D-}

galactopyranosyl]- $\alpha$-D-glucopyranoside (13): A solution of $12(50.4 \mathrm{mg}, 0.0909$ mmol), 8 (49 mg, $0.0967 \mathrm{mmol}), N$-(thiophenyl)-e-caprolactam (1) $(22.1 \mathrm{mg}, 0.0999$ mmol), tri-tert-butylpyrimidine (TTBP) $(96 \mathrm{mg}, 0.3871 \mathrm{mmol}$ ) and flame activated AW300 molecular sieves were stirred in dry dichloromethane $(2 \mathrm{ml})$ for $0.5 \mathrm{~h}$ at ambient temperature. The reaction mixture was then cooled to $-0{ }^{\circ} \mathrm{C}$ (Ice Water), stirred for $5 \mathrm{~min}$ and then followed by injection of trifluoromethanesulfonic anhydride $(19 \mu \mathrm{L}, 0.1123$ mmol). The reaction mixture was stirred for $10 \mathrm{~min}$ at $0{ }^{\circ} \mathrm{C}$, filtered and then concentrated. The crude mixture was directly purified by silica gel flash column chromatography (hexane/EtOAc, 4:1) to afford $\mathbf{1 3}(81 \mathrm{mg}, 95 \%)$ as a white solid/residue. 13: ${ }^{1} \mathrm{H}$ NMR $\left(500 \mathrm{MHz}, \mathrm{CDCl}_{3}\right) \delta$ 8.12-8.09 (m, 2H), 8.01-7.95 (m, 4H), 7.64-7.11 (m, 24H), $6.22(\mathrm{t}, 1 \mathrm{H}, J=9.4 \mathrm{~Hz}), 5.36(\mathrm{~s}, 1 \mathrm{H}), 5.30\left(\mathrm{~d}, 1 \mathrm{H}, J=3.5 \mathrm{~Hz}, \mathrm{H}^{\prime}-1\right), 5.21(\mathrm{dd}, 1 \mathrm{H}$, $J=10.3,3.5 \mathrm{~Hz}), 5.16(\mathrm{~d}, 1 \mathrm{H}, J=3.8 \mathrm{~Hz}, \mathrm{H}-1)), 4.82(\mathrm{dd}, 1 \mathrm{H}, J=12.3,1.9 \mathrm{~Hz}), 4.63$ $\left(\mathrm{ABq}, 2 \mathrm{H}, v_{\mathrm{A}}=4.65, v_{\mathrm{B}}=4.61, J_{\mathrm{AB}}=12.1 \mathrm{~Hz}\right), 4.49(\mathrm{dd}, 1 \mathrm{H}, J=12.2,4.0 \mathrm{~Hz}), 4.33(\mathrm{~d}$, $1 \mathrm{H}, J=12.1 \mathrm{~Hz}), 4.31(\mathrm{~d}, 1 \mathrm{H}, J=9.0 \mathrm{~Hz}), 4.23(\mathrm{ddd}, 1 \mathrm{H}, J=10.1,3.8,1.8 \mathrm{~Hz}), 4.19-$ $4.10(\mathrm{~m}, 3 \mathrm{H}), 3.98-3.88(\mathrm{~m}, 3 \mathrm{H}), 3.79(\mathrm{dd}, 1 \mathrm{H}, J=12.8,1.5 \mathrm{~Hz}), 3.42(\mathrm{~s}, 3 \mathrm{H}) ;{ }^{13} \mathrm{C} \mathrm{NMR}$ (125 MHz, $\left.\mathrm{CDCl}_{3}\right)$ 166.2, 166.0, 165.4, 138.6, 138.2, 137.6, 133.4, 133.3, 133.0, 129.9, $129.7,129.6,129.5,129.0,128.8,128.6,128.4,128.3,128.2,128.1,128.07,128.0,127.7$, 
127.5, 127.4, 126.2, 100.7, 99.6 (C'-1), 96.8 (C-1), 75.9, 74.3, 74.2, 74.0, 73.3, 72.3, 72.07, 69.2, 68.4, 63.7, 63.2, 55.4, 29.6; HRMS (MALDI-FTMS) $\mathrm{m} / \mathrm{z}$ : $(\mathrm{M}+\mathrm{Na})^{+}$calc for $\mathrm{C}_{55} \mathrm{H}_{52} \mathrm{O}_{14} 959.3249$, found 959.3225 .
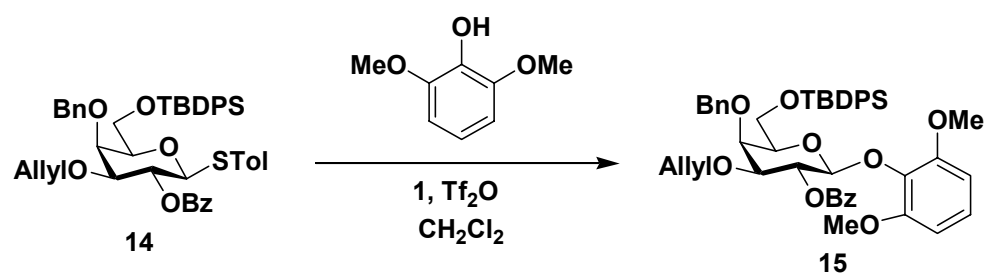

\section{2-O-Benzoyl-3-O-allyl-4-O-benzyl-6-O-tert-butyldiphenylsilyl-1-O-(2,6-O-}

dimethoxyphenyl)- $\beta$-D-galactopyranoside (15): A solution of 14 (51 $\mathrm{mg}, 0.0672$

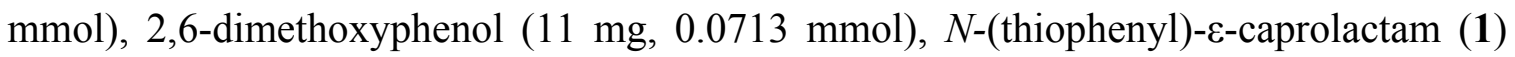
(16 mg, $0.0723 \mathrm{mmol}$ ), and flame activated AW-300 molecular sieves were stirred in dry dichloromethane $(2 \mathrm{ml})$ for $0.5 \mathrm{~h}$ at ambient temperature. The reaction mixture was then cooled to $-45^{\circ} \mathrm{C}\left(\mathrm{CH}_{3} \mathrm{CN} /\right.$ dry ice $)$, stirred for $5 \mathrm{~min}$ and then followed by injection of trifluoromethanesulfonic anhydride $(14 \mu \mathrm{L}, 0.0832 \mathrm{mmol})$. The reaction mixture was stirred for $0.5 \mathrm{~h}$ at $-45{ }^{\circ} \mathrm{C}$, then at ambient temperature for $0.5 \mathrm{~h}$. The solution was quenched with triethylamine, filtered and concentrated. The crude mixture was directly purified by silica gel flash column chromatography (hexane/EtOAc, 6:1) to afford 15 (45 $\mathrm{mg}, 85 \%$ ) as a white solid/residue.

15: ${ }^{1} \mathrm{H}$ NMR $\left(500 \mathrm{MHz}, \mathrm{CDCl}_{3}\right) \delta$ 8.20-8.15 (m, 2H), 7.71-7.60 (m, 4H), 7.59-7.53 (m, 1H), 7.49-7.23 (m, 13H), $6.91(\mathrm{t}, 1 \mathrm{H}, J=8.5 \mathrm{~Hz}), 6.40(\mathrm{~d}, 2 \mathrm{H}, J=8.7 \mathrm{~Hz}), 6.07-5.90(\mathrm{~m}$, $1 \mathrm{H}), 5.66-5.60(\mathrm{~m}, 1 \mathrm{H}), 5.39-5.31(\mathrm{~m}, 1 \mathrm{H}), 5.19-5.14(\mathrm{~m}, 1 \mathrm{H}), 5.12(\mathrm{~d}, 1 \mathrm{H}, J=11.0 \mathrm{~Hz})$, 4.83 (dd, 1H, $J=8.8,5.2 \mathrm{~Hz}), 4.67$ (d, 1H, $J=11.1 \mathrm{~Hz}), 4.43-4.31$ (m, 3H), 4.0 (t, 1H, $J$ $=9.2 \mathrm{~Hz}), 3.83(\mathrm{br} \mathrm{s}, 2 \mathrm{H}), 3.72(\mathrm{dd}, 1 \mathrm{H}, J=9.2,5.4 \mathrm{~Hz}), 3.36(\mathrm{~s}, 6 \mathrm{H}), 1.12(\mathrm{~s}, 9 \mathrm{H}) ;{ }^{13} \mathrm{C}$ NMR (125 MHz, $\left.\mathrm{CDCl}_{3}\right)$ 166.3, 153.4, 138.9, 135.7, 135.6, 135.0, 134.4, 133.4, 133.2, 
$132.7,130.7,129.8,129.7,128.2,128.1,127.7,127.2,125.8,124.3,116.5,104.6(\mathrm{C}-1)$, 98.7, 76.5, 75.1, 74.9, 71.9, 71.8, 71.5, 61.7, 55.3, 26.9, 19.3; HRMS (MALDI-FTMS) $m / z:(\mathrm{M}+\mathrm{Na})^{+}$calc for $\mathrm{C}_{47} \mathrm{H}_{52} \mathrm{O}_{9} \mathrm{Si}$ 811.3273, found 811.3276.

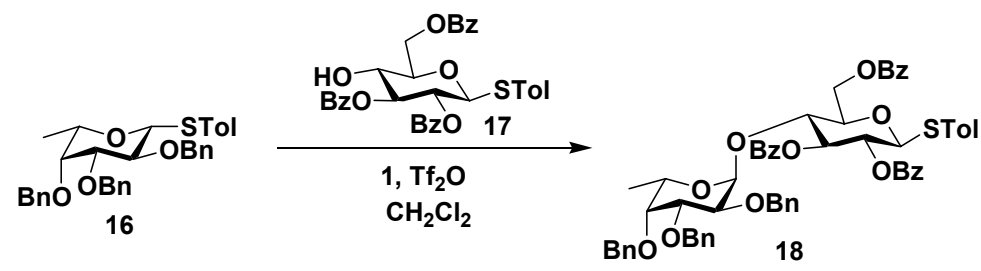

$p$-Methylphenyl-2,3,6-tri- $O$-benzoyl-4- $O$-(2,3,4-tri- $O$-benzyl- $\alpha$-L-fucopyranosyl)-1thio- $\beta$-D-glucopyranoside (18): A solution of $16(50 \mathrm{mg}, 0.0925 \mathrm{mmol}), 17(56 \mathrm{mg}$, $0.0935 \mathrm{mmol}), N$-(thiophenyl)-E-caprolactam (1) $(22 \mathrm{mg}, 0.0994 \mathrm{mmol})$, and flame activated AW-300 MS were stirred in dry dichloromethane $(2 \mathrm{ml})$ for $1 \mathrm{~h}$ at ambient temperature. Trifluoromethanesulfonic anhydride $(19 \mu \mathrm{L}, 0.1129 \mathrm{mmol})$ was then injected into the solution. The reaction mixture was stirred at ambient temperature until all the starting materials were consumed. The solution was quenched with triethylamine $(150 \mu \mathrm{L})$, filtered and concentrated. The crude mixture was directly purified by silica gel flash column chromatography (hexane/EtOAc, 5:1) to afford $\mathbf{1 8}(67 \mathrm{mg}, 84 \%)$ as a white foam. (higher yields $>95 \%$ can be accomplished using procedure for 19). Spectral data was consistent with published data on $\mathbf{1 8} .^{1}$
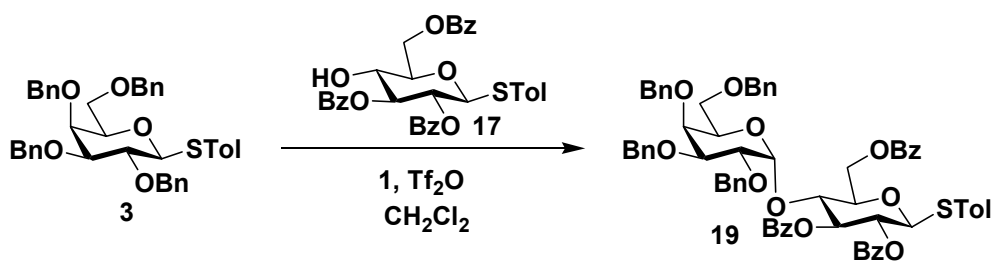

$p$-Methylphenyl-2,3,6-tri- $O$-benzoyl-4- $O$-(2,3,4-tri- $O$-benzyl- $\alpha$-D-galactopyranosyl)

\footnotetext{
${ }^{1}$ Mong, T. K.-K.; Lee, H.-K.; Durón, S.G.; Wong, C.-H. Proc. Natl. Acad. Sci. U.S.A. 2003, 100, 797-802.
} 
-1-thio- $\beta$-D-glucopyranoside (19): A solution of 3 (26.1 mg, $0.0404 \mathrm{mmol}), 17$ (21.3 mg, $0.0356 \mathrm{mmol}), \mathrm{N}$-(thiophenyl)-e-caprolactam (1) (12 mg, $0.0542 \mathrm{mmol})$, and flame activated AW-300 MS were stirred in dry dichloromethane $(2 \mathrm{ml})$ for $0.5 \mathrm{~h}$ at ambient temperature. The reaction mixture was then cooled to $-78{ }^{\circ} \mathrm{C}$ (acetone/Dry Ice), stirred for $5 \mathrm{~min}$ and then followed by injection of trifluoromethanesulfonic anhydride $(13 \mu \mathrm{L}$, $0.0773 \mathrm{mmol}$ ). The reaction mixture was stirred until the cold bath reached $-10{ }^{\circ} \mathrm{C}$ and then was quenched with triethylamine $(100 \mu \mathrm{L})$, filtered and concentrated. The crude mixture was directly purified by silica gel flash column chromatography (hexane/EtOAc, 5:1) to afford $\mathbf{1 9}(37 \mathrm{mg}, 93 \%)$ as a white foam.

19: ${ }^{1} \mathrm{H}$ NMR $\left(500 \mathrm{MHz}, \mathrm{CDCl}_{3}\right) \delta$ 8.04-7.98 (m, 2H), 7.95-7.86 (m, 4H), $7.61(\mathrm{t}, 1 \mathrm{H}, J=$ $7.0 \mathrm{~Hz}), 7.62-7.08(\mathrm{~m}, 30 \mathrm{H}), 6.88(\mathrm{~d}, 2 \mathrm{H}, J=8.0 \mathrm{~Hz}), 5.83(\mathrm{t}, 1 \mathrm{H}, J=9.4 \mathrm{~Hz}), 5.33(\mathrm{t}$, $1 \mathrm{H}, J=9.9 \mathrm{~Hz}), 4.99$ (d, 1H, $\left.J=3.4 \mathrm{~Hz}, \mathrm{H}^{\prime}-1\right), 4.92(\mathrm{dd}, 1 \mathrm{H}, J=12.3,1.8 \mathrm{~Hz}), 4.85$ (d, $1 \mathrm{H}, J=9.8 \mathrm{~Hz}, \mathrm{H}-1), 4.75(\mathrm{~d}, 1 \mathrm{H}, J=12.0 \mathrm{~Hz}), 4.60-4.50(\mathrm{~m}, 1 \mathrm{H}), 4.60(\mathrm{~d}, 2 \mathrm{H}, J=2.3$ $\mathrm{Hz}), 4.42(\mathrm{~d}, 1 \mathrm{H}, J=11.3 \mathrm{~Hz}),), 4.60\left(\mathrm{ABq}, 2 \mathrm{H}, v_{\mathrm{A}}=4.29, v_{\mathrm{B}}=4.20, J_{\mathrm{AB}}=11.8 \mathrm{~Hz}\right)$, 4.26-4.14 (m, 2H), 4.00-3.91 (m, 3H), 3.91-3.80 (m, 1H), $3.85(\mathrm{dd}, 1 \mathrm{H}, J=10.3,2.6 \mathrm{~Hz})$, $3.77(\mathrm{dd}, 1 \mathrm{H}, J=10.0,3.3 \mathrm{~Hz}), 3.39(\mathrm{~m}, 2 \mathrm{H}), 2.23(\mathrm{~s}, 3 \mathrm{H}) ;{ }^{13} \mathrm{C} \mathrm{NMR}\left(125 \mathrm{MHz}, \mathrm{CDCl}_{3}\right)$ $165.9,1.65 .5,1.65 .2,138.7,138.4,138.2,137.9,133.9,133.2,133.1,133.0,129.9,129.8$, $129.7,129.6,129.5,129.3,128.4,128.3,128.2,128.2,128.1,128.0,127.9,127.8,127.6$, 127.5, 127.4, 127.3, 99.5 (C'-1), 85.7 (C-1), 78.6, 77.4, 75.8, 75.2, 75.0, 74.9, 74.7, 73.3, 73.1, 72.9, 70.7, 70.6, 68.6, 63.4, 21.2; HRMS (MALDI-FTMS) $m / z:(\mathrm{M}+\mathrm{Na})^{+}$calc for $\mathrm{C}_{68} \mathrm{H}_{64} \mathrm{O}_{13} \mathrm{~S} 1143.396$, found 1143.3978. 


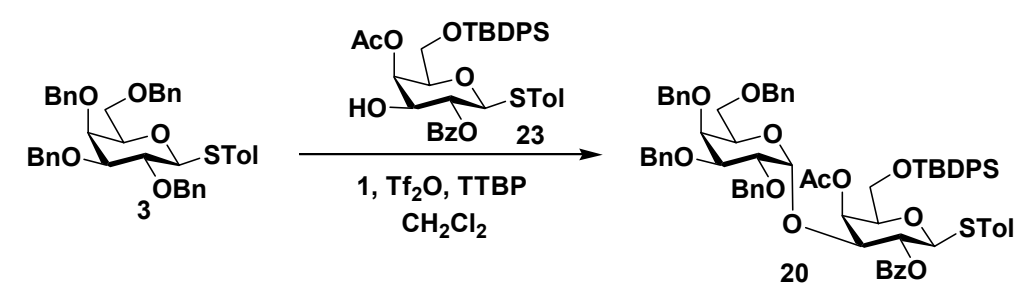

p-Methylphenyl-2-O-benzoyl-3- $O$-[2,3,4,6-tetra- $O$-benzyl- $\alpha$-D-galactopyranosyl]-4$O$-acetyl-6- $O$-tert-butyldiphenylsilyl-1-thio- $\beta$-D-galactopyranoside (20): A solution of 3 (25.3 mg, $0.0391 \mathrm{mmol}), 23$ (25.4 mg, $0.0379 \mathrm{mmol}), N$-(thiophenyl)- $\varepsilon$-caprolactam (1) (9.1 mg, $0.0411 \mathrm{mmol})$, ), tri-tert-butylpyrimidine (TTBP) $(21.4 \mathrm{mg}, 0.0857 \mathrm{mmol})$ and flame activated AW-300 MS were stirred in dry dichloromethane $(2 \mathrm{ml})$ for $0.5 \mathrm{~h}$ at ambient temperature. Trifluoromethanesulfonic anhydride $(8 \mu \mathrm{L}, 0.0473 \mathrm{mmol})$ was then injected into the reaction mixture at ambient temperature and stirred for $5 \mathrm{~min}$. The solution was stirred for $0.5 \mathrm{~h}$ at ambient temperature then filtered and concentrated. The crude mixture was directly purified by silica gel flash column chromatography (hexane/EtOAc, 6:1) to afford $20(33 \mathrm{mg}, 73 \%)$ as a colorless oil.

20: ${ }^{1} \mathrm{H}$ NMR $\left(500 \mathrm{MHz}, \mathrm{CDCl}_{3}\right) \delta$ 8.20-7.98 (m, 2H), 7.67-7.62 (m, 4H), 7.49-7.21 (m, 29H), 7.14-7.09 (m, 2H), $7.03(\mathrm{~d}, 2 \mathrm{H}, J=8.2 \mathrm{~Hz}), 5.63(\mathrm{~d}, 1 \mathrm{H}, J=3.0 \mathrm{~Hz}), 5.51(\mathrm{t}, 1 \mathrm{H}$, $J=9.8 \mathrm{~Hz}), 5.26\left(\mathrm{~d}, 1 \mathrm{H}, J=3.0 \mathrm{~Hz}, \mathrm{H}^{\prime}-1\right), 4.76-4.70(\mathrm{~m}, 2 \mathrm{H}), 4.63\left(\mathrm{ABq}, 2 \mathrm{H}, \mathrm{v}_{\mathrm{A}}=\right.$ $\left.4.65, v_{\mathrm{B}}=4.60, J_{\mathrm{AB}}=11.9 \mathrm{~Hz}\right), 4.61(\mathrm{~d}, 1 \mathrm{H}, J=11.8 \mathrm{~Hz}, \mathrm{H}-1), 4.51(\mathrm{~d}, 1 \mathrm{H}, J=11.8 \mathrm{~Hz})$, $4.35(\mathrm{dd}, 2 \mathrm{H}, J=11.8,2.4 \mathrm{~Hz}), 4.28(\mathrm{~d}, 1 \mathrm{H}, J=11.5 \mathrm{~Hz}), 4.10(\mathrm{dd}, 1 \mathrm{H}, J=9.7,2.8 \mathrm{~Hz})$, $3.91(\mathrm{dd}, 1 \mathrm{H}, J=10.0,3.3 \mathrm{~Hz}), 3.86(\mathrm{t}, 1 \mathrm{H}, J=6.0 \mathrm{~Hz}), 3.77(\mathrm{dd}, 1 \mathrm{H}, J=9.5,5.6 \mathrm{~Hz})$, 3.67-3.60 (m, 2H), $3.51(\mathrm{dd}, 1 \mathrm{H}, J=9.8,2.8 \mathrm{~Hz}), 3.45(\mathrm{dd}, 1 \mathrm{H}, J=9.5,7.0 \mathrm{~Hz}), 3.17-$ $3.14(\mathrm{~m}, 2 \mathrm{H}), 2.30(\mathrm{~s}, 3 \mathrm{H}), 1.74(\mathrm{~s}, 3 \mathrm{H}), 1.04(\mathrm{~s}, 9 \mathrm{H}) ;{ }^{13} \mathrm{C} \mathrm{NMR}\left(125 \mathrm{MHz}, \mathrm{CDCl}_{3}\right)$ $170.0,164.9,138.8,138.7,138.4,138.3,137.7,135.6,133.2,133.0,132.9,132.4,129.9$, $129.88,129.84,129.79,129.72,129.6,128.4,128.3,128.2,128.18,128.11,128.0,127.9$, 
127.74, 127.71, 127.56, 127.50, 127.4, 127.3, 127.29, 127.21, 93.0 (C'-1), 87.5 (C-1), $78.8,77.8,75.7,74.9,74.4,73.3,73.2,73.1,72.8,69.8,69.6,69.3,64.9,62.2,26.8,21.1$, 20.4. 19.1; HRMS (MALDI-FTMS) $m / z$ : $(\mathrm{M}+\mathrm{Na})^{+}$calc for $\mathrm{C}_{72} \mathrm{H}_{76} \mathrm{O}_{12} \mathrm{SSi} 1215.4719$, found 1215.4694.

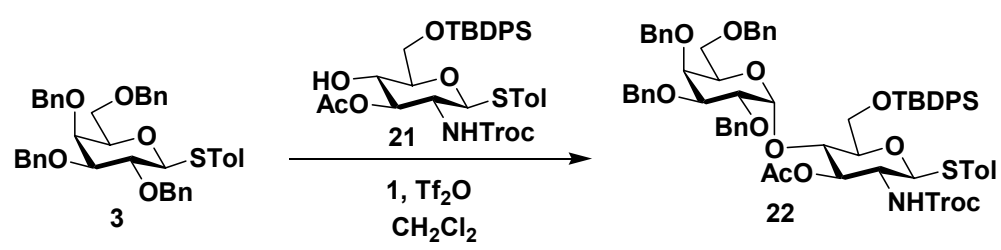

$p$-Methylphenyl-2-deoxy-2-(2,2,2-trichloro-ethoxylcarbonylamino)-3-O-acetyl-4-O-

[2,3,4,6-tetra- $O$-benzyl- $\alpha$-D-galactopyranosyl]-6-O-tert-butyldiphenylsilyl-1-thio- $\beta$ -

D-glucopyranoside (22): A solution of 3 (100 mg, $0.1546 \mathrm{mmol}), 21(111 \mathrm{mg}, 0.1498$

mmol), $N$-(thiophenyl)- $\varepsilon$-caprolactam (1) $(37 \mathrm{mg}, 0.1672 \mathrm{mmol})$, and flame activated AW-300 MS were stirred in dry dichloromethane $(2 \mathrm{ml})$ for $0.5 \mathrm{~h}$ at ambient temperature. The reaction mixture was then cooled to $-45^{\circ} \mathrm{C}\left(\mathrm{CH}_{3} \mathrm{CN} /\right.$ Dry Ice), stirred for $5 \mathrm{~min}$ and then followed by injection of trifluoromethanesulfonic anhydride $(30 \mu \mathrm{L}, 0.1783 \mathrm{mmol})$. The reaction mixture was stirred for $0.5 \mathrm{~h}$ at $-45^{\circ} \mathrm{C}$, then warmed over $2 \mathrm{~h}$ to $-20^{\circ} \mathrm{C}$. The solution was quenched with triethylamine, filtered and concentrated. The crude mixture was directly purified by silica gel flash column chromatography (hexane/EtOAc, 5:1) to afford $22(138 \mathrm{mg}, 71 \%)$ as a pale yellow oil.

22: ${ }^{1} \mathrm{H}$ NMR $\left(500 \mathrm{MHz}, \mathrm{CDCl}_{3}\right) \delta$ 7.72-7.66 (m, 4H), 7.36-7.23 (m, 26H), 7.13-7.11 (m, 2H), $7.04(\mathrm{~d}, 2 \mathrm{H}, J=8.0 \mathrm{~Hz}), 6.0(\mathrm{~d}, 1 \mathrm{H}, J=8.9 \mathrm{~Hz}), 5.16(\mathrm{t}, 1 \mathrm{H}, J=7.0 \mathrm{~Hz}), 5.02(\mathrm{~d}$, $\left.1 \mathrm{H}, J=3.2 \mathrm{~Hz}, \mathrm{H}^{\prime}-1\right), 4.89(\mathrm{~m}, 2 \mathrm{H}), 4.78\left(\mathrm{ABq}, 2 \mathrm{H}, v_{\mathrm{A}}=4.81, v_{\mathrm{B}}=4.76, J_{\mathrm{AB}}=12.3 \mathrm{~Hz}\right)$, 4.75-4.65 (m, 3H), $4.66(\mathrm{~d}, 1 \mathrm{H}, J=10.1 \mathrm{~Hz}, \mathrm{H}-1), 4.53(\mathrm{~d}, 1 \mathrm{H}, J=11.3 \mathrm{~Hz}), 4.25(\mathrm{ABq}$, $\left.2 \mathrm{H}, v_{\mathrm{A}}=4.29, v_{\mathrm{B}}=4.21, J_{\mathrm{AB}}=11.7 \mathrm{~Hz}\right), 4.10-4.02(\mathrm{~m}, 2 \mathrm{H}), 3.99(\mathrm{dd}, 1 \mathrm{H}, J=10.4,3.5$ 
$\mathrm{Hz}), 3.95-3.88(\mathrm{~m}, 1 \mathrm{H}), 3.87-3.77(\mathrm{~m}, 3 \mathrm{H}), 3.74(\mathrm{dd}, 1 \mathrm{H}, J=10.2,2.5 \mathrm{~Hz}), 3.68-3.63(\mathrm{~m}$, 1H), $3.40(\mathrm{t}, 1 \mathrm{H}, J=7.4 \mathrm{~Hz}), 3.32(\mathrm{dd}, 1 \mathrm{H}, J=9.0,5.8 \mathrm{~Hz}), 2.32(\mathrm{~s}, 3 \mathrm{H}), 1.81(\mathrm{~s}, 3 \mathrm{H})$, $1.06(\mathrm{~s}, 9 \mathrm{H}) ;{ }^{13} \mathrm{C}$ NMR $\left(125 \mathrm{MHz}, \mathrm{CDCl}_{3}\right) 170.4,154.2,138.6,138.5,137.9,137.8$, 137.6, 135.8, 135.7, 133.3, 133.0, 132.2, 130.1, 129.6, 138.5, 128.4, 128.3, 128.2, 128.1, 127.9, 127.8, 127.7, 127.6, 127.4, 98.5 (C'-1), 95.5 (C-1), 87.1, 80.4, 78.2, 75.7, 74.7, 74.6, 73.7, 73.6, 73.4, 72.8, 70.3, 68.5, 63.6, 54.8, 29.7, 26.9, 21.1, 20.8. 19.3, 14.2;

HRMS (MALDI-FTMS) $m / z$ : $(\mathrm{M}+\mathrm{Na})^{+}$calc for $\mathrm{C}_{68} \mathrm{H}_{74} \mathrm{Cl}_{3} \mathrm{NO}_{12} \mathrm{SSi} 1284.3659$, found 1284.3562 .

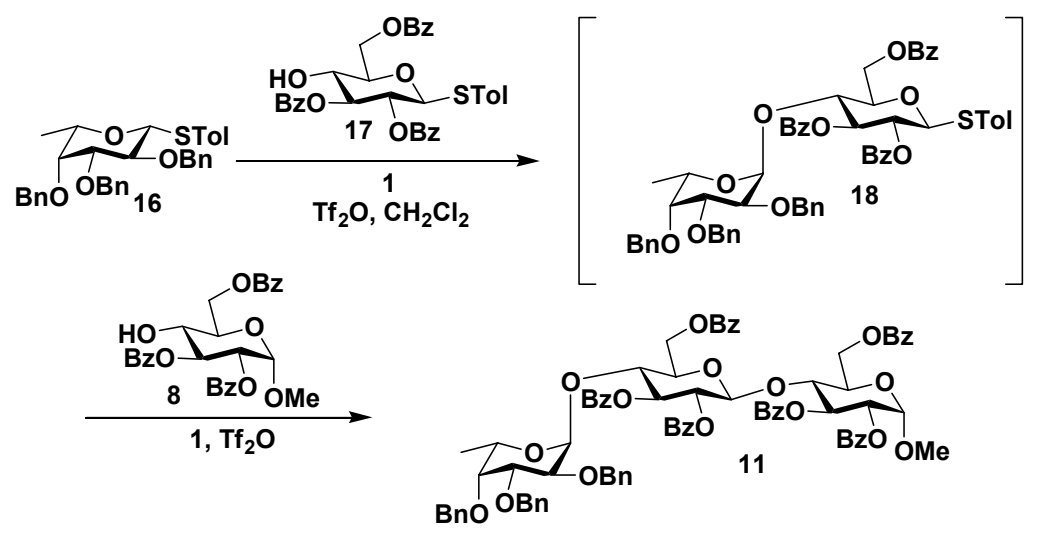

\section{Methyl-2,3,6-tri- $O$-benzoyl-4- $O$-[2,3,6-tri- $O$-benzoyl-4- $O$ - $\{2,3,4-$ tri- $O$-benzyl- $\alpha$-L-}

fucopyranosyl\}- $\beta$-D-glucopyranosyl]- $\alpha$-D-glucopyranoside (11): A solution of 16 (42

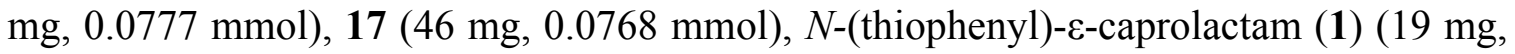
$0.0858 \mathrm{mmol})$, and flame activated AW-300 MS were stirred in dry dichloromethane (2 $\mathrm{ml}$ ) for $1 \mathrm{~h}$ at ambient temperature. The reaction mixture was then cooled to $-45{ }^{\circ} \mathrm{C}$ $\left(\mathrm{CH}_{3} \mathrm{CN} /\right.$ Dry Ice), stirred for $5 \mathrm{~min}$ and then followed by injection of trifluoromethanesulfonic anhydride $(15 \mu \mathrm{L}, 0.0892 \mathrm{mmol})$ and then warmed to ambient temperature. The formation of $\mathbf{1 8}$ was monitored by TLC (hexanes:EtOAc 3:1). After the starting materials were consumed, $8(39 \mathrm{mg}, 0.0770 \mathrm{mmol})$ and $N$-(thiophenyl)- $\varepsilon$ caprolactam (1) (56 mg, $0.2530 \mathrm{mmol})$ were added to the reaction mixture and stirred for 
$30 \mathrm{~min}$. The solution was cooled to $-45^{\circ} \mathrm{C}$ and then trifluoromethanesulfonic anhydride (45 $\mu \mathrm{L}, 0.2675 \mathrm{mmol}$ ) was injected into the mixture, stirred for $0.5 \mathrm{~h}$, then stirred at ambient temperature $0.5 \mathrm{~h}$ (the reaction was stirred overnight, but did not see increase in yield by tlc). The solution was quenched with triethylamine, filtered and concentrated. The crude mixture was directly purified by silica gel flash column chromatography (hexane/EtOAc, gradient eluent 5:1 to 3:1) to afford 11 (39 $\mathrm{mg}, 36 \%$ ) as a colorless oil. 11: ${ }^{1} \mathrm{H}$ NMR $\left(600 \mathrm{MHz}, \mathrm{CDCl}_{3}\right) \delta$ 8.05-7.09 (m, 45H), $6.02(\mathrm{t}, 1 \mathrm{H}, J=9.1 \mathrm{~Hz}), 5.57(\mathrm{t}$, $1 \mathrm{H}, J=9.4 \mathrm{~Hz}), 5.33(\mathrm{dd}, 1 \mathrm{H}, J=9.9,8.0 \mathrm{~Hz}), 5.12-5.09(\mathrm{~m}, 1 \mathrm{H}), 5.07(\mathrm{~d}, 1 \mathrm{H}, J=3.7$ Hz, H-1), 4.87 (d, 1H, J = 7.9 Hz, H'-1), 4.79-4.66 (m, 5H), 4.62 (d, 1H, $J=9.2 \mathrm{~Hz})$, $4.60(\mathrm{~d}, 1 \mathrm{H}, J=5.6 \mathrm{~Hz}, \mathrm{H}$ '-1), 4.58-4.55 (m, 2H), 4.46-4.42 (m, 1H), 4.45 (d, 1H, $J=$ $11.4 \mathrm{~Hz}), 4.15-4.06(\mathrm{~m}, 4 \mathrm{H}), 3.85-3.77(\mathrm{~m}, 2 \mathrm{H}), 3.66$ (ddd, $1 \mathrm{H}, J=10.0,5.1,1.9 \mathrm{~Hz})$, $3.62(\mathrm{q}, 1 \mathrm{H}, J=6.3 \mathrm{~Hz}), 3.33(\mathrm{~s}, 3 \mathrm{H}), 0.55$ (d, $3 \mathrm{H}, J=6.3 \mathrm{~Hz}) ;{ }^{13} \mathrm{C}$ NMR $(150 \mathrm{MHz}$, $\left.\mathrm{CDCl}_{3}\right) 165.9,165.8,165.7,165.3,164.9,138.7,138.3,138.2,133.3,133.1,132.9$, $129.9,129.8,129.7,129.5,129.4,128.5,128.4,128.3,128.2,128.0,127.5,127.4,100.5$ (C'-1), 100.1 (C'”-1), 96.7 (C-1), 79.1, 77.6, 76.4, 75.7, 75.4, 74.7, 74.3, 73.9, 73.9, 72.7, $72.4,72.2,70.0,68.4,67.5,62.8,62.4,55.4,29.7,15.8$; HRMS (MALDI-FTMS) $m / z$ : $(\mathrm{M}+\mathrm{Na})^{+}$calc for $\mathrm{C}_{82} \mathrm{H}_{76} \mathrm{O}_{21}$ 1419.4771, found 1419.4761. 